\title{
Tumor Effects on Cerebral White Matter as Characterized by Diffusion Tensor Tractography
}

\author{
Corie W. Wei, Gang Guo, David J. Mikulis
}

\begin{abstract}
Background: Diffusion tensor MRI fiber tractography (DTT) is the first non-invasive in vivo technique for delineating specific white matter (WM) tracts. In cerebral neoplasm, DTT can be used to illustrate the relationship of the tumor with respect to adjacent WM trajectories. Methods: Fiber tractography was used in this study to assess tumor-induced changes in WM trajectories in three cases of cerebral neoplasm: glioblastoma multiforme, meningioma, and anaplastic astrocytoma. Results: Three patterns of WM alteration were identified: 1) disruption, 2) displacement, and 3) infiltration. Tumor disruption of WM tracts was observed in glioblastoma multiforme, which terminated fibers crossing the corpus callosum. In meningioma, DTT illustrated bulk displacement of the corticospinal tract in the affected hemisphere as well as preservation of the deviated axons. In anaplastic astrocytoma, fiber tracking demonstrated disruption of WM tracts at the tumor origin as well as intact axons through areas of tumor infiltration. Conclusions: Fiber tracking results correlated with the clinical and histopathological features of the tumor. Larger case series will be required to determine if fiber tracking can add accuracy to existing imaging methods for grading tumors.
\end{abstract}

RÉSUMÉ: Effet de masse de tumeurs sur la substance blanche cérébrale évalué par IRM en tenseur de diffusion. Contexte : La tractographie par IRM en tenseur de diffusion (TTD) pour étudier les fibres de la substance blanche (SB) est la première technique non effractive in vivo permettant de localiser des faisceaux spécifiques. Dans les tumeurs cérébrales, la TTD peut être utilisée pour illustrer la relation entre la tumeur et la trajectoire des faisceaux de la SB adjacents à la tumeur. Méthodes : La tractographie de faisceaux de fibres a été utilisée dans cette étude pour évaluer les changements induits par la tumeur dans la trajectoire de faisceaux de la SB chez trois cas de néoplasie cérébrale : un glioblastome multiforme, un méningiome et un astrocytome anaplasique. Résultats : Trois types de changements ont été identifiés dans la SB : 1) interruption; 2) déplacement et 3) infiltration. L' interruption de faisceaux de la SB à travers le corps calleux a été observée dans le glioblastome multiforme. Dans le méningiome, la TTD a montré un déplacement en masse du faisceau corticospinal dans l'hémisphère touché ainsi que la préservation des axones déplacés. Dans l'astrocytome anaplasique, la tractographie a montré une interruption des faisceaux de la SB là où la tumeur avait pris naissance ainsi que des axones intacts dans les zones d'infiltration de la tumeur. Conclusions : Les résultats de la tractographie concordaient avec les manifestations cliniques et histopathologiques de la tumeur. Il faudra étudier un plus grand nombre de cas pour déterminer si la tractographie de fibres peut améliorer la précision des méthodes d'imagerie actuelles pour évaluer le grade d'une tumeur.

Can. J. Neurol. Sci. 2007; 34: 62-69

Diffusion tensor magnetic resonance imaging (DTI) is the first and only non-invasive technique capable of delineating white matter (WM) pathways in vivo. ${ }^{1}$ Diffusion tensor magnetic resonance imaging can provide qualitative and quantitative information about the directionality (anisotropy) and magnitude (diffusivity) of water diffusion along a vector in 3-dimensional space. ${ }^{2-5}$ The success of diffusion imaging is related to the fact that during their random, diffusion-driven displacements, water molecules probe tissue structures at a microscopic scale well beyond conventional imaging resolution. ${ }^{6}$ In WM fiber tracts, organized bundles of axonal membranes and myelin sheaths present substantial barriers to diffusion, especially in directions perpendicular to that of the fibers. These barriers render water diffusion highly anisotropic (directionally dependent) along the longitudinal direction of the axons. ${ }^{7}$

By taking advantage of the unique directionality of water, diffusion tensor tractography (DTT) can generate realistic white

\footnotetext{
From the Schulich School of Medicine and Dentistry (CWW), University of Western Ontario, London, ON; Department of Medical Imaging (DJM), Toronto Western

Hospital, University of Toronto, Toronto, ON, Canada; Department of Radiology (GG), Shantou University, Shantou, China.

ReCEIVEd July 10, 2006. ACCEPTED IN FINAL FORM November 23, 2006.

Reprint requests to: David J. Mikulis, Toronto Western Hospital, Department of Medical Imaging, Room 3MC-431, 399 Bathurst Street, Toronto, Ontario, L6J 7P9, Canada.
} 
matter tract trajectories in three dimensions. ${ }^{8-11}$ Diffusion tensor tractography allows for voxel-by-voxel mapping of local white matter tracts using the assumption that the eigenvector associated with the largest eigenvalue is aligned with the direction of the fiber bundle..$^{8,12-14}$ In studies of healthy human brains, DTT maps show high concordance with established atlases of brain anatomy. ${ }^{15,16}$

Many brain tumors originate within and propagate through cerebral white matter. A cerebral neoplasm may affect WM trajectories at either the cellular or gross anatomic level. ${ }^{17}$ Some tumors may alter the location and/or direction of adjacent WM tracts through bulk mass displacement while leaving axons intact. Other tumors may infiltrate between individual fibers without disrupting their directional organization. Complete destruction of a WM tract from tumor invasion is also possible. However, conventional MR techniques (including T1-weighted, T2-weighted, and FLAIR imaging) cannot give any detailed information about the integrity and location of WM tracts. On the other hand, functional MRI can assess the effects of brain tumors in the cerebral cortex while providing little information about connective pathways in the WM. ${ }^{18,19}$

Diffusion tensor tractography provides a new and unique opportunity for studying tumor-related alterations of neuronal pathways. Previous investigations in patients with spaceoccupying lesions have employed fiber tractography to illustrate displacement of the corticospinal tract (CST), superior longitudinal fasciculus, and corona radiata. ${ }^{20-22}$ In addition, the precise characterization of the relationship between tumor and WM tracts is useful for planning target-focused tumor therapy, as it can facilitate the preservation of eloquent fiber tracts. Many authors have reported on the clinical application of DTT in preoperative planning ${ }^{5,23-26}$ and postoperative assessment. ${ }^{27}$

In this article, we provide a pictorial review of changes in fiber tract trajectories adjacent to brain tumors using DTT. We have also attempted to categorize the appearances of tumoraltered WM tracts into three patterns: disruption, displacement, and infiltration.

\section{METHODS}

\section{Patient Information}

We evaluated three patients with intracranial neoplasms using diffusion tensor tractography. Patient One was a 70-year-old male with glioblastoma multiforme (GBM) involving the corpus callosum. Neurological symptoms at the time of diagnosis were significant for episodic vertigo and confusion. Patient Two was a 62-year-old male with meningioma and no associated neurological deficits. Patient Three was a 69-year-old female with diffuse anaplastic astrocytoma (Grade III/IV) involving the left internal capsule and brainstem. Neurological deficits at the time of diagnosis included confusion, complete palsy of the left oculomotor nerve, left eye blindness, and right-sided hemiparesis. All diagnoses were based on the histological features of specimens obtained from a stereotactic biopsy according to the World Health Organization classification.

\section{Imaging Protocol}

All studies were performed in accordance with the institutional committee guidelines on human research. Patients underwent the routine MR protocol (including $\mathrm{T}_{1}, \mathrm{~T}_{2}$, DWI, and FLAIR) for the evaluation of brain tumors after giving informed consent. The DTI was performed at the end of the routine examination before gadolinium administration. The total imaging time for DTI was three minutes. All routine MRI and DTI scans were performed using a 3.0 T MRI system (Signa EXCITE, GE HealthCare, Milwaukee, WI) equipped with an 8channel RF head-coil (MR imaging devices). A single-shot, multirepetition echo planar imaging sequence with diffusion gradients applied in 25 directions was used for DTI: TR 10,000 ms, TE $84 \mathrm{~ms}$, matrix 128 x 128, field of view $240 \mathrm{~mm}^{2}, 5 \mathrm{~mm}$ thickness, $0 \mathrm{~mm}$ gap, voxel dimension $1.8 \times 1.8 \times 5 \mathrm{~mm}, b$ factor $1000 \mathrm{~s} / \mathrm{mm}^{2}$.

\section{Data processing}

All image post processing was performed on the scanner console using a subprogram of the Functool ${ }^{\mathrm{TM}}$ image analysis software (Version 3.1.22, General Electric Medical Systems, Buc, France). Image misregistration from motion distortion was corrected in an automated procedure using Functool. The fractional anisotropy (FA) index is a rotationally invariant scalar index of anisotropy that scales from 0 (isotropic medium) to 1 (maximum anisotropy). The scalar values of FA for each pixel were used to encode the colors on the FA maps with red representing highly anisotropic water diffusion and blue representing isotropy.

\section{Tractography}

Fiber tracking was performed by using fiber assignment in the continuous tracking algorithm. ${ }^{11}$ The tracking algorithm was initiated from a user-defined "seed" region of interest (ROI). Tracking was performed in both anterograde and retrograde directions according to the direction of the principal eigenvector in the ROI. Diffusion tensors, eigenvalues, and eigenvectors were computed from linearly interpolated diffusion tensor images at each iteration. The propagation was terminated when the tract trajectory reached a voxel with FA less than 0.2 (the estimated major eigenvector direction becomes less accurate as FA decreases and is very sensitive to image noise for FA less than 0.2) or when the angle between two consecutive steps was greater than $45^{\circ}$. We used two schemes to localize the structures of interest: (1) defining a single seed ROI and displaying all the trajectories that passed through this ROI; (2) defining a "seed" and "target" ROI and displaying all the trajectories passing through both of these regions. ${ }^{9}$

To visualize the effects of tumor on neighboring WM tracts, we targeted two major WM trajectories: the corticospinal tract and fibers crossing the corpus callosum. In this study, the corticospinal tract incorporated all fibers originating along the motor cortex, which converged through the corona radiata and posterior limb of the internal capsule on their way to the lateral funiculus of the spinal cord. ${ }^{28}$ The ROIs were determined on the $\mathrm{T}_{2}$-weighted EPI scans and were automatically transferred onto the coregistered FA and MD maps. To visualize the CST, we placed the "seed" ROI in the posterior limb of the internal capsule and the "target" ROI at the level of the ipsilateral pons. The CST in the hemisphere with tumor involvement was reconstructed and compared with the CST in the intact hemisphere. For reconstruction of fibers crossing the corpus 


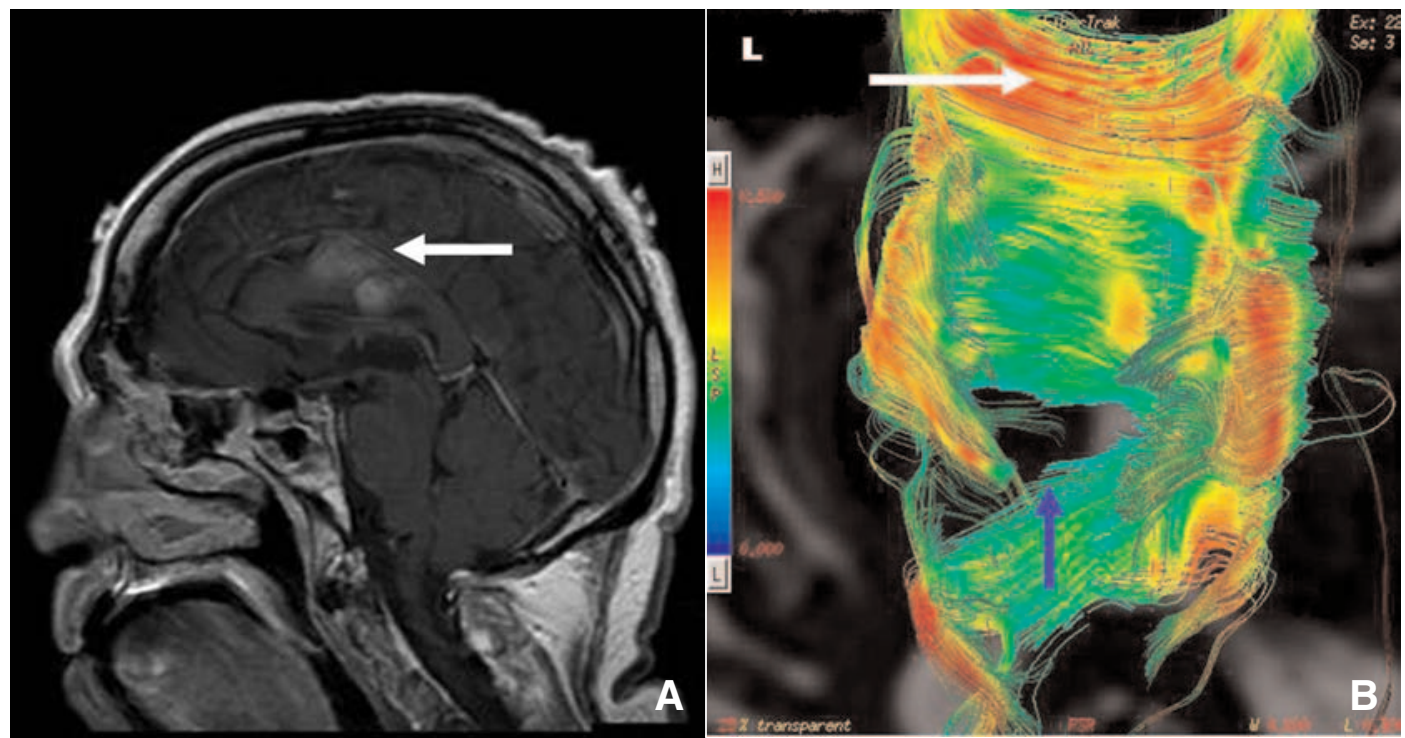

Figure 1: WM tract disruption as a result of GBM. (a) Sagittal T1-weighted image with gadolinium enhancement shows the GBM in the left parasagittal frontal lobe with invasion into the body of the corpus callosum (arrow). (b) Tractography map (top view) shows disruption of fibers crossing the corpus callosum (blue arrow) in a region corresponding to the location of the tumor compared to the normal white matter tracts (white arrow). The left side of the image corresponds to the left side of the subject.

callosum, we placed a single "seed" ROI in the commissure of corpus callosum in the transverse plane.

Fiber trajectories were displayed with colors encoded by the FA value at each voxel. The color scale on the tractography map corresponded to fractional anistropy with red representing highly anisotropic water diffusion and blue representing isotropy. It is of interest to note that tractograms are not displayed according to the conventions of clinical radiology. In the top view and the posterior coronal view of the DTT maps, the left side of the tractography map corresponds to the left side of the subject; similarly, the right side of the map corresponds to the right side of the subject.

\section{RESUlts}

\section{Pattern of Tumor Disruption of WM Tracts: Patient One}

In the case of glioblastoma multiforme, DTT illustrated abrupt termination of fibers crossing the corpus callosum in the vicinity of the tumor (Figure 1).

\section{Pattern of Tumor Displacement of WM Tracts: Patient Two}

Fiber tracking illustrated displacement of the corticospinal tract as a result of tumor mass effect in the case of meningioma (Figure 2). Bulk displacement by the tumor changed the location and orientation of the CST in the affected hemisphere without affecting the coherence of fiber bundle.
Pattern of Tumor Infiltration and Disruption of WM Tracts: Patient Three

In the case of anaplastic astrocytoma, axial FLAIR showed presence of the tumor in the left pons with extension into the genu of left internal capsule (Figures $3 a, b)$. It is of interest to note that the tumor had benign appearance on conventional MRI and did not enhance with gadolinium.

The FA maps showed marked reduced anisotropy in tumorinvolved regions in the left pons and internal capsule compared to the contralateral side (Figures 3c, d). For fiber tracking purposes, the seed ROI was placed in the tumor-involved left pons (Figure 3c); the target ROI was placed in the tumorinvolved posterior limb of the left internal capsule (Figure 3d). Region of interests were placed in the same locations on the contralateral side.

The tractography map illustrated the CST through tumorinfiltrated regions between the internal capsule and the pons without changes in location or orientation (Figure 3e). In addition, the colors of the tractography map demonstrated reduced FA in the tumor-involved CST as compared to the contralateral side. However, the tractography map showed abrupt termination of fibers of the left CST at the level of the pons, possibly as a result of tumor destruction.

\section{DisCUSSION}

Diffusion tensor tractography is the only imaging modality 
capable of generating realistic 3-D maps of WM trajectories in vivo. Our study showed that tractography was effective in probing microstructural abnormalities in the WM in the presence of cerebral neoplasms, showing three patterns of involvement: disruption, displacement, and infiltration.

In the case of GBM, disruption of WM fibers was illustrated in the region corresponding to the tumor location. This pattern of neuronal disruption on tractography may represent WM tract destruction in the context of a highly malignant tumor. Proliferation of high-grade astrocytic tumors in the white matter is associated with destruction of tissue and cell structures. The loss of WM fiber organization can diminish the anisotropy of water diffusion to below the threshold level required for the fiber-tracking algorithm, thus resulting in termination of WM trajectories near tumor boundaries. However, DTT did not allow us to pinpoint the exact location of tumor margins since these are histologically indistinct for infiltrating tumors.

Although WM destruction is a likely explanation for the appearance of fiber termination on tractography, it is important to recognize that DTT is an indirect measure of white matter integrity and that a variety of explanations for changes in FA and termination of the fiber tracking algorithm are possible. The fiber tracking algorithm dictates that a fiber trajectory is terminated when the FA of a voxel is below 0.2 or when the angle between two consecutive steps was greater than $45^{\circ}$. As a result, a dramatically displaced fiber tract $\left(>45^{\circ}\right)$ in the pathway of tumor progression cannot be visualized by tractography. In addition, fluid shifts related to cerebral edema provides another explanation for fiber termination. It is not clear how the fiber tracking results are affected by vigorous edema in peritumoral regions. Vasogenic edema may result in a relative increase in perpendicular diffusion as compared to parallel diffusion and was found to be associated with decreased $\mathrm{FA}^{29}$. If significant anisotropy reduction is present, it may no longer be possible to detect sufficient diffusion directionality for reconstructing tracts despite the presence of intact axonal structures ${ }^{25}$. As a result, peritumoral edema may prevent the precise localization and characterization of WM fibers in close proximity to tumors.

Diffusion tensor tractography illustrated displacement of intact neuronal tracts in the case of meningioma. We expect similar effects of fiber tract displacement in extra-axial tumors as well as any non-invasive, non-infiltrating expansile parenchymal lesion.

In the case of anaplastic astrocytoma, WM changes on the tractography map were a mixed pattern of tumor infiltration and disruption. In tumor-involved regions between the internal capsule and the pons, the CST displayed reduced anisotropy without changes in location or orientation. This suggests that DTT may be able to resolve structurally intact axonal pathways within the tumor volume. Although anaplastic astrocytoma is a high-grade tumor, neoplastic cells can infiltrate between fibers without disrupting the neurons at early stages of tumor invasion, resulting in reduced anisotropy of water diffusion along the axons. At the level of the pons, however, the tractography map showed abrupt termination of fibers of the left CST, possibly as a result of tumor destruction. Disruption of the left CST on DTT correlated with clinical symptoms of the patient, who had left cranial nerve III palsy and right-sided hemiparesis at the time of diagnosis. Neoplastic cells may have originated in the pons, thus destroying the neuronal fibers at the pons while infiltrating between axons along the pathway of proliferation. Based on the assumption that the aggressiveness of the tumor is related to the site of origin, DTT can potentially illustrate the pathway of tumor progression. Since this tumor had benign appearance on conventional MRI and did not enhance with gadolinium, DTTillustrated tumor effect on neighboring neuronal tracts provided additional information on the malignant nature of the tumor and

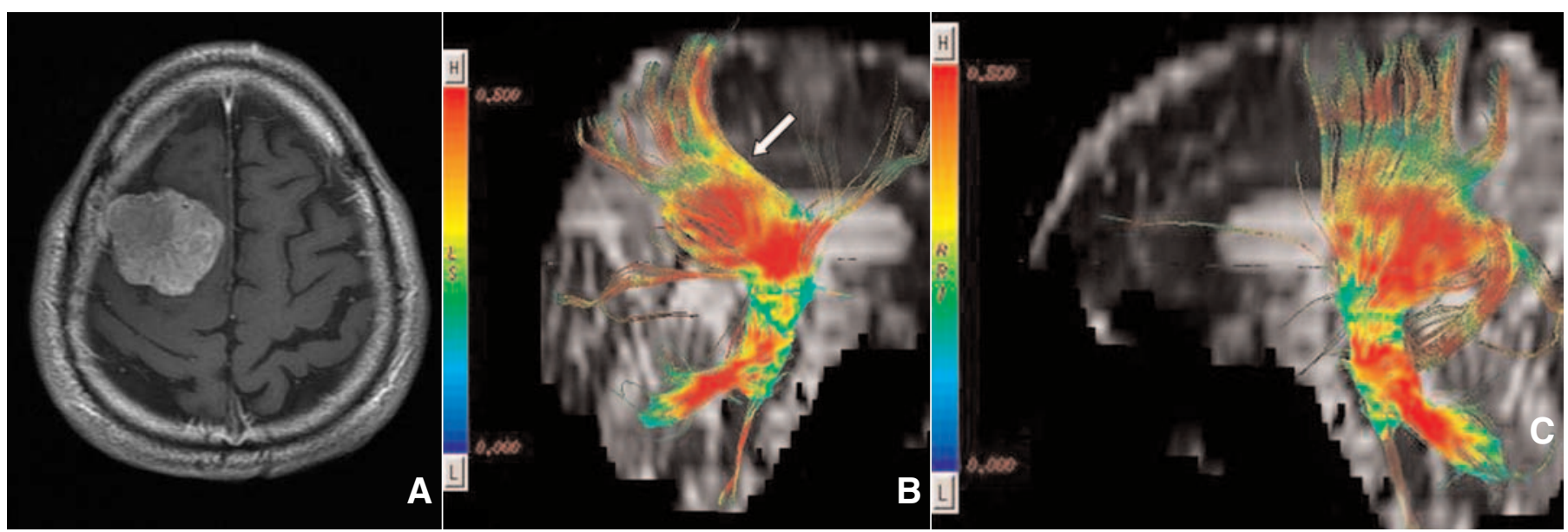

Figure 2: WM tract displacement by meningioma. (a) Axial T1-weighted image with gadolinium enhancement shows the meningioma in right frontal lobe. b) Tractography map (right sagittal view) shows displacement of the right CST by the tumor (arrow) compared with the normal CST on the contralateral side (Figure c). 


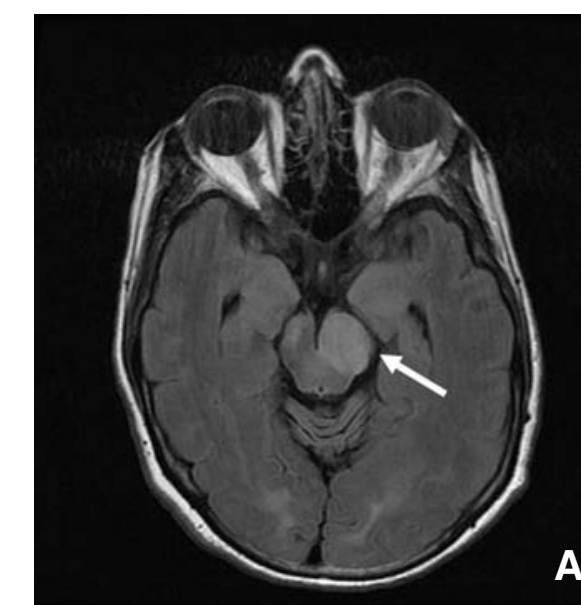

A
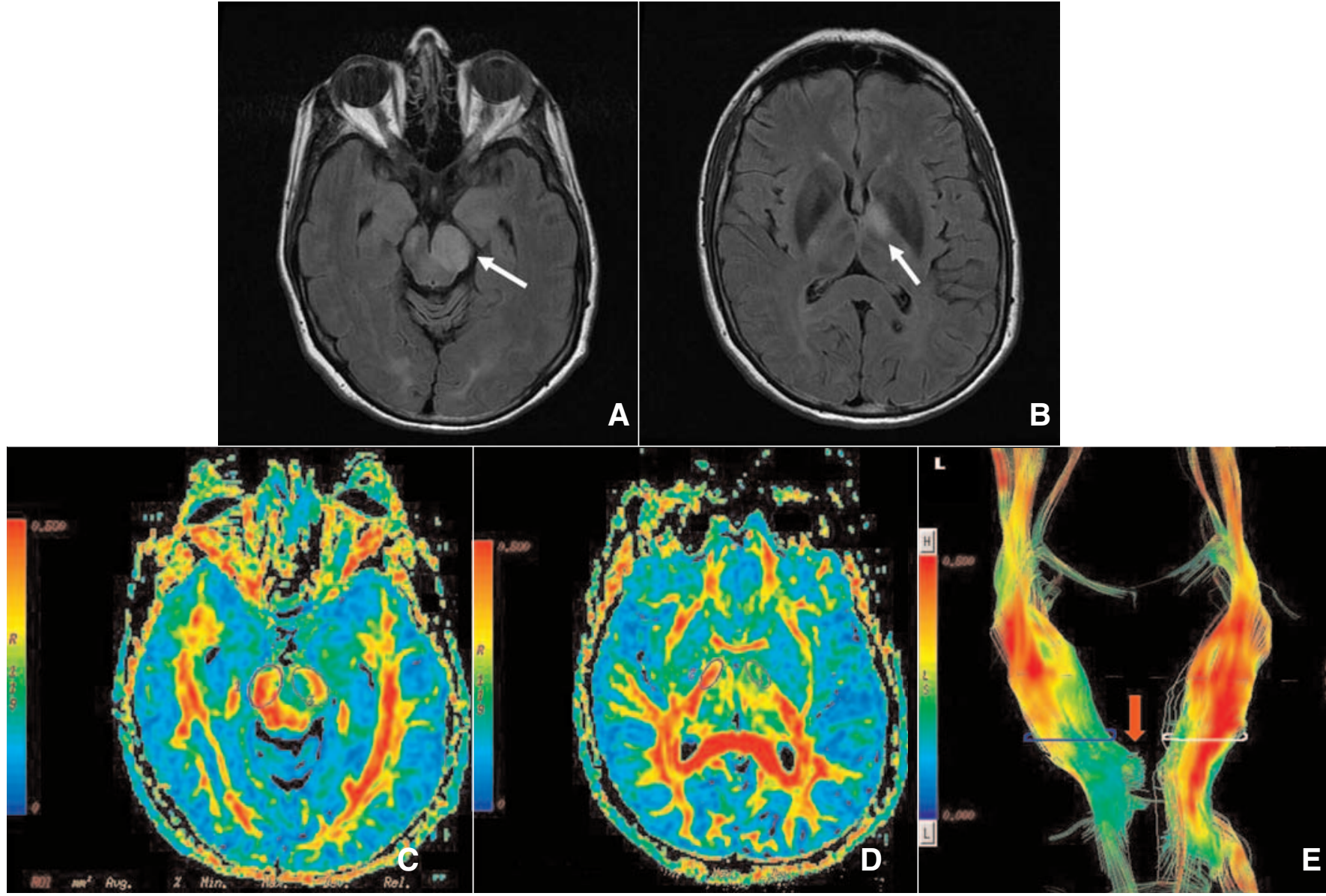

Figure 3: WM tracts infiltration and disruption by anaplastic astrocytoma. ( $a, b)$ Axial FLAIR shows the tumor arising from the left pons extending into the genu of the left internal capsule and basal ganglia (arrows). (c) FA map. The FA of tumor-involved left pons is markedly reduced compared to the contralateral side. Seed ROIs for fiber tracking are placed on bilateral pons. (d) FA map. The FA of tumor-involved left internal capsule is markedly reduced compared to the ride side. Target ROIs are placed in tumor-involved posterior limb of the left internal capsule and corresponding area on right side. (e) Tractography map (posterior coronal view) shows left CST passing through the tumor. The left CST through areas of tumor infiltration shows reduced FA (circled in blue) compared to intact CST on the contralateral side (circled in white). At the level of the pons, fibers of the left CST are terminated as a result of tumor disruption (red arrow).

thus enhanced the power of radiological diagnosis of tumor pathology.

Our study findings indicate that DTT can provide radiological evidence of tumor pathology based on the interaction between the tumor itself and local white matter tracts. However, studies with larger case series are needed to validate tractography results with clinical and histopathological data. In particular, neuronal tract alterations in the vicinity of benign tumors should be further characterized with DTT.

In the clinical application of DTI to assess brain tumors, much of previous research has been focused on employing DTI parameters for tissue characterization. ${ }^{30}$ Studies have found an inverse relationship between tumor cellularity and mean diffusivity. ${ }^{31-34}$ There is some evidence that intratumoral anisotropy is inversely related to tumor grade in gliomas ${ }^{35}$ while others have reported contradictory findings. ${ }^{36}$ Decreased anisotropy was also found to be slightly correlated with cranial nerve deficits in pontine tumors. ${ }^{37}$ In addition, qualitative DTI data such as the directionally encoded color maps have been used to characterize the effect of tumors on local fiber tracts. Field et al has described four distinct patterns of tumor-altered WM tracts on FA-weighted colored directionality maps: displacement, infiltration, peritumoral edema, and disruption. ${ }^{29}$ In terms of neurosurgical planning and assessment, studies have reported on the utility of fiber tractography in guiding tumor resections and preserving clinically eloquent fiber tracts..$^{5,23-26}$ In a recent study, Schonberg et al used an fMRI-based seed ROI selection approach to produce a more comprehensive mapping of functional neuronal pathways. ${ }^{38}$ In the future, DTI maybe combined with functional MRI maps to provide additional imaging information about tumors and their effects on neuronal pathways prior to resection. 
In a previous study using directional diffusivity as a noninvasive marker of underlying pathology, it was found that a reduction in parallel diffusivity $(\lambda \|)$ reflected axonal degeneration while decreased perpendicular diffusivity $(\lambda \perp)$ was consistent with myelin degeneration. ${ }^{39}$ In the case of GBM, we expect that foci of tumor necrosis and axonal destruction would be associated with nearly equal water diffusivities in all directions and a markedly reduced $\lambda \|$. On the other hand, areas of tumor infiltration in the case of anaplastic astrocytoma would be characterized by increased $\lambda \perp$ as a result of the dispersion of the axons. However, eigenvalues were not available in this study to validate these hypotheses.

Our study suggests fiber tractography has the potential to provide greater specificity to the pathological diagnosis of cerebral tumors on imaging. In a recent study, Roberts et al introduced a quantitative application of fiber tracking in tissue characterization by measuring the density of WM fibers in the vicinity of high-grade gliomas using the "fiber density index" (FDi). ${ }^{40}$ However, the relationship between FDi and tumor histopathology has not been established.

While the 3-D tract reconstruction provides a new way to evaluate WM architecture, its limitations should also be recognized. First, the diffusion tensor used to measure the fiber tract direction is a voxel-averaged quantity. If voxels contain anistropic fibrous tissue with a uniform fiber direction, the eigenvector associated with the largest eigenvalue of the effective diffusion tensor will provide an unbiased estimate of microscopic fiber field direction vector. ${ }^{4}$ However, if there is a non-uniform distribution of fiber directions within a voxel, the principal eigenvector only corresponds to a consensus average of fibers within this voxel. Problems arise especially at junctions of fiber crossing, merging, or branching. In these situations, the accuracy of the reconstructed WM trajectories is questionable.

Finally, fiber tracking is a user-defined process. Standardized ROI locations and adequate threshold values for the tracking algorithm are essential for achieving an objective and uniform fiber tracking result. ${ }^{41}$ In particular, tractography maps are highly dependent on the threshold values of FA and trajectory angles for the termination of tracking. Although tract termination on DTT may reflect tumor-induced axonal damage, an alternative explanation is that neoplastic glial cells may have caused enough structural disorganization to reduce the anisotropy to subthreshold levels. In the latter case, the fiber tractography may fail to delineate structurally intact axons. On the other hand, relaxing the "stopping" criteria may allow an algorithm to proceed through low-anisotropy regions, but this increases the risk of generating spurious results because estimates of primary eigenvector direction become less reliable for low-anisotropy tensors. ${ }^{42,43}$ However, our findings suggest that DTT can resolve structurally intact axons through the tumor volume. Tract disruption on DTT also correlated with neurological deficits. In addition, a recent pilot study reported excellent correlation between preoperative and postoperative DTI of the pyramidal tract and clinical motor function. ${ }^{44}$ Future investigations with larger samples are warranted to define and validate the optimal thresholds for fiber tracking algorithms.

In conclusion, we have demonstrated the application of 3-D DTT to the study of tumor-induced alterations in the white matter architecture. The 3-D reconstruction of WM pathways can not only add to the diagnostic power of tumor imaging but also enhance the specificity of tumor therapy. With future refinement and validation of the fiber tracking technique, DTT can be exploited in the clinical assessment and therapeutic planning for cerebral neoplasm.

\section{ACKNOWLEDGEMENT}

This research was supported by funding from the Institute of Medical Science Summer Student Program. The authors thank the patients for their participation.

\section{REFERENCES}

1. Wimberger DM, Roberts TP, Barkovich AJ, Prayer LM, Moseley ME, Kucharczyk J. Identification of "premyelination" by diffusion-weighted MRI. J Comput Assist Tomography. 1995; 19:28-33.

2. Brunberg JA, Chenevert TL, McKeever PE, Ross DA, Junck LR, Muraszko KM, et al. In vivo MR determination of water diffusion coefficients and diffusion anisotropy: correlation with structural alteration in gliomas of the cerebral hemispheres. AJNR Am J Neuroradiol. 1995;16:361-71.

3. Chien D, Kwong KK, Gress DR, Buonanno FS, Buxton RB, Rosen BR. MR diffusion imaging of cerebral infarction in humans. AJNR Am J Neuroradiol. 1992;13:1097-1102; discussion 11031095.

4. Pierpaoli C, Jezzard P, Basser PJ, Barnett A, Di Chiro G. Diffusion tensor MR imaging of the human brain. Radiology. 1996;201:637-48.

5. Witwer BP, Moftakhar R, Hasan KM, Deshmukh P, Haughton V, Field A, et al. Diffusion-tensor imaging of white matter tracts in patients with cerebral neoplasm. J Neurosurg. 2002;97:568-75.

6. Le Bihan D, Mangin JF, Poupon C, Clark CA, Pappata S, Molko N, et al. Diffusion tensor imaging: concepts and applications. J Magn Reson Imaging. 2001;13:534-46.

7. Bammer R. Basic principles of diffusion-weighted imaging. Eur $\mathbf{J}$ Radiol. 2003;45:169-84.

8. Basser PJ, Pajevic S, Pierpaoli C, Duda J, Aldroubi A. In vivo fiber tractography using DT-MRI data. Magn Reson Med. 2000;44: 625-32.

9. Conturo TE, Lori NF, Cull TS, Akbudak E, Snyder AZ, Shimony JS, et al. Tracking neuronal fiber pathways in the living human brain. Proc Natl Acad Sci USA. 1999;96:10422-7.

10. Gossl C, Fahrmeir L, Putz B, Auer LM, Auer DP. Fiber tracking from DTI using linear state space models: detectability of the pyramidal tract. Neuroimage. 2002;16:378-88.

11. Mori S, Crain BJ, Chacko VP, van Zijl PC. Three-dimensional tracking of axonal projections in the brain by magnetic resonance imaging. Ann Neurol. 1999;45:265-9.

12. Bammer R, Acar B, Moseley ME. In vivo MR tractography using diffusion imaging. Eur J Radiol. 2003;45:223-34.

13. Lori NF, Akbudak E, Shimony JS, Cull TS, Snyder AZ, Guillory RK, et al. Diffusion tensor fiber tracking of human brain connectivity: aquisition methods, reliability analysis and biological results. NMR Biomed. 2002;15:494-515.

14. Mori S, van Zijl PC. Fiber tracking: principles and strategies - a technical review. NMR Biomed. 2002;15:468-80.

15. Catani M, Howard RJ, Pajevic S, Jones DK. Virtual in vivo interactive dissection of white matter fasciculi in the human brain. Neuroimage. 2002;17:77-94.

16. Mamata H, Mamata Y, Westin CF, Shenton ME, Kikinis R, Jolesz FA, et al. High-resolution line scan diffusion tensor MR imaging of white matter fiber tract anatomy. AJNR Am J Neuroradiol. 2002;23:67-75.

17. Field AS, Alexander AL, Wu YC, Hasan KM, Witwer B, Badie B. Diffusion tensor eigenvector directional color imaging patterns in the evaluation of cerebral white matter tracts altered by tumor. Top Magn Reson Imaging. 2004;15:315-24. 
18. Cosgrove GR, Buchbinder BR, Jiang H. Functional magnetic resonance imaging for intracranial navigation. Neurosurg Clin $\mathrm{N}$ Am. 1996;7:313-22.

19. Schulder M, Maldjian JA, Liu WC, Holodny AI, Kalnin AT, Mun IK, et al. Functional image-guided surgery of intracranial tumors located in or near the sensorimotor cortex. J Neurosurg. 1998; 89:412-18.

20. Mori S, Frederiksen K, van Zijl PC, Stieltjes B, Kraut MA, Solaiyappan M, et al. Brain white matter anatomy of tumor patients evaluated with diffusion tensor imaging. Ann Neurol. 2002;51:377-80.

21. Holodny AI, Ollenschleger MD, Liu WC, Schulder M, Kalnin AJ. Identification of the corticospinal tracts achieved using bloodoxygen-level-dependent and diffusion functional MR imaging in patients with brain tumors. AJNR Am J Neuroradiol. 2001; 22:83-8.

22. Field AS, Wu YC, Alexander AL. Principal diffusion direction in peritumoral fiber tracts: color map patterns and directional statistics. Ann N Y Acad Sci. 2005;1064:193-201.

23. Moller-Hartmann W, Krings T, Coenen VA, Mayfrank L, Weidemann J, Kranzlein H, et al. Preoperative assessment of motor cortex and pyramidal tracts in central cavernoma employing functional and diffusion-weighted magnetic resonance imaging. Surg Neurol. 2002;58:302-7; discussion 308.

24. Holodny AI, Schwartz TH, Ollenschleger M, Liu WC, Schulder M. Tumor involvement of the corticospinal tract: diffusion magnetic resonance tractography with intraoperative correlation. J Neurosurg. 2001;95:1082.

25. Clark CA, Barrick TR, Murphy MM, Bell BA. White matter fiber tracking in patients with space-occupying lesions of the brain: a new technique for neurosurgical planning? Neuroimage. 2003; 20:1601-8.

26. Jena R, Price SJ, Baker C, Jefferies SJ, Pickard JD, Gillard JH, et al. Diffusion tensor imaging: possible implications for radiotherapy treatment planning of patients with high-grade glioma. Clin Oncol (R Coll Radiol). 2005;17:581-90.

27. Yu CS, Li KC, Xuan Y, Ji XM, Qin W. Diffusion tensor tractography in patients with cerebral tumors: a helpful technique for neurosurgical planning and postoperative assessment. Eur J Radiol. 2005;56:197-204.

28. Jellison BJ, Field AS, Medow J, Lazar M, Salamat MS, Alexander AL. Diffusion tensor imaging of cerebral white matter: a pictorial review of physics, fiber tract anatomy, and tumor imaging patterns. AJNR Am J Neuroradiol. 2004;25:356-69.

29. Field AS. Diffusion tensor imaging at the crossroads: fiber tracking meets tissue characterization in brain tumors. AJNR Am J Neuroradiol. 2005;26:2168-9.

30. Field AS. Diffusion tensor imaging at the crossroads: fiber tracking meets tissue characterization in brain tumors. AJNR Am J Neuroradiol. 2005;26:2168-9.
31. Sugahara T, Korogi Y, Kochi M, Ikushima I, Shigematu Y, Hirai T, et al. Usefulness of diffusion-weighted MRI with echo-planar technique in the evaluation of cellularity in gliomas. J Magn Reson Imaging. 1999;9:53-60.

32. Gauvain KM, McKinstry RC, Mukherjee P, Perry A, Neil JJ, Kaufman BA, et al. Evaluating pediatric brain tumor cellularity with diffusion-tensor imaging. AJR Am J Roentgenol. 2001; $177: 449-54$

33. Kono K, Inoue Y, Nakayama K, Shakudo M, Morino M, Ohata K, et al. The role of diffusion-weighted imaging in patients with brain tumors. AJNR Am J Neuroradiol. 2001;22:1081-8.

34. Guo AC, Cummings TJ, Dash RC, Provenzale JM. Lymphomas and high-grade astrocytomas: comparison of water diffusibility and histologic characteristics. Radiology. 2002;224:177-83.

35. Inoue $\mathrm{T}$, Ogasawara K, Beppu $\mathrm{T}$, Ogawa A, Kabasawa H. Diffusion tensor imaging for preoperative evaluation of tumor grade in gliomas. Clin Neurol Neurosurg. 2005;107:174-80.

36. Lu S, Ahn D, Johnson G, Law M, Zagzag D, Grossman RI. Diffusion-tensor MR imaging of intracranial neoplasia and associated peritumoral edema: introduction of the tumor infiltration index. Radiology. 2004;232:221-8.

37. Helton KJ, Phillips NS, Khan RB, Boop FA, Sanford RA, Zou P, et al. Diffusion tensor imaging of tract involvement in children with pontine tumors. AJNR Am J Neuroradiol. 2006;27:786-93.

38. Schonberg T, Pianka P, Hendler T, Pasternak Ofer, Assaf Y. Characterization of displace white matter by brain tumors using combined DTI and fMRI. Neuroimage. 2006;30:1100-11.

39. Song SK, Sun SW, Ju WK, Lin SJ, Cross AH, and Neufeld AH. Diffusion tensor imaging detects and differentiates axon and myelin degeneration in mouse optic nerve after retinal ischemia. Neuroimage. 2003;20 (3): 1714-22.

40. Roberts TP, Liu F, Kassner A, Mori S, Guha A. Fiber density index correlates with reduced fractional anisotropy in white matter of patients with glioblastoma. AJNR Am J Neuroradiol. 2005; 26:2183-6.

41. Lee SK, Kim DI, Kim J, Kim DJ, Kim HD, Kim DS, et al. Diffusion-tensor MR imaging and fiber tractography: a new method of describing aberrant fiber connections in developmental CNS anomalies. Radiographics. 2005;25:53-65; discussion 66-58.

42. Basser PJ, Pajevic S. Statistical artifacts in diffusion tensor MRI (DT-MRI) caused by background noise. Magn Reson Med. 2000; 44:41-50.

43. Wu YC, Field AS, Chung MK, Badie B, Alexander AL. Quantitative analysis of diffusion tensor orientation: theoretical framework. Magn Reson Med. 2004;52:1146-55.

44. Laundre BJ, Jellison BJ, Badie B, Alexander AL, Field AS. Diffusion tensor imaging of the corticospinal tract before and after mass resection as correlated with clinical motor findings: preliminary data. AJNR Am J Neuroradiol. 2005; 26:791-6. 